\title{
Tool Readiness for TEM
}

\author{
Silvia Aerts
}

Thermo Fisher Scientific, Eindhoven, Netherlands

Corresponding author: silvia.aerts@thermofisher.com

Wouldn't it be much easier if the microscope is just ready to go instead of scheduling downtime for (automated) microscope alignments requiring expert knowledge? Tool Readiness offers exactly this to its users, ease-of-use and high productivity.

The purpose of Tool Readiness is to support a specific application. Based on what the application plans to do (TEM, STEM, EDS), Tool Readiness knows which alignments are important for this application job and which can be skipped. Only the ones important for this job are checked. Nothing more, nothing less. The application calls Tool Readiness just before starting the job.

Each Tool Readiness run consists of three steps:

1. Check if the alignment is still valid. The previous result is compared with the current microscope state. When these results differ, the alignment will run to verify the current status. Besides this, every alignment has its own expiration time and will run when this period passed. The process capability index (Cpk) of each alignment and known day to day microscope drift determine the expiration time. The $\mathrm{Cpk}$ is the ratio of the alignment sensitivity for optimal performance and the accuracy of the automated alignment.

2. Perform a measurement to check the current alignment status. Alignment is only needed when the result is outside the acceptance criteria which is based on the Cpk. Tool Readiness navigates automatically to the correct sample position with the aid of AI enhanced template matching. Measurements run in vacuum, amorphous area or an area with structure and details depending on the measurement demands; a crystalline silicon area is used to check the distortion and magnification accuracy.

3. Execute the alignment and verify its correctness with a measurement. If the result is within the acceptance criteria, the alignment is saved, and a new expiration period starts. Image template matching is used to minimize the shift between microscope modes and second order probe corrector aberrations get corrected with a new optimized routine.

With this new approach, Tool Readiness guarantees the application executes a flawless job, resulting in HR images and metrology accuracy. 